$9-7-2021$

\title{
Atención de la salud sexual y reproductiva de mujeres migrantes: Un mapeo de actores de la sociedad civil en Chiapas, México-2020
}

Silvana Larrea-Schiavon

Population Council

Lucía Vázquez Quesada

Population Council

Esperanza Basurto-Alcalde

Natalia Polgovsky

Isabel Vieitez Martínez

Population Council

See next page for additional authors

Follow this and additional works at: https://knowledgecommons.popcouncil.org/departments_sbsr-rh How does access to this work benefit you? Let us know!

\section{Recommended Citation}

Larrea-Schiavon, Silvana, Lucía Vázquez-Quesada, Esperanza Basurto-Alcalde, Natalia Polgovsky, Isabel Vieitez Martínez, and Pooja Sripad. 2021. "Atención de la salud sexual y reproductiva de mujeres migrantes: Un mapeo de actores de la sociedad civil en Chiapas, México-2020." México: Population Council. 


\section{Authors}

Silvana Larrea-Schiavon, Lucía Vázquez Quesada, Esperanza Basurto-Alcalde, Natalia Polgovsky, Isabel Vieitez Martínez, and Pooja Sripad 


\section{Atención de la salud sexual y}

reproductiva de mujeres migrantes: Un mapeo de actores de la sociedad civil en Chiapas, México

\section{0}






\title{
P. POPULATION
}

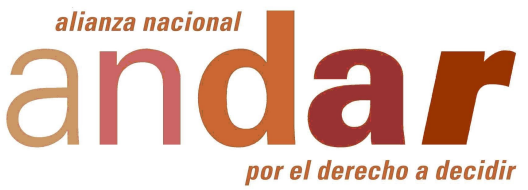

\begin{abstract}
Atención de la salud sexual y reproductiva de mujeres migrantes: Un mapeo de actores de la sociedad civil en Chiapas, México. 2020.
\end{abstract}

\section{Equipo de investigación:}

Protocolo de investigación, análisis de datos, redacción y/o revisión de informe:

Silvana Larrea-Schiavon* Lucía Vázquez-Quesada* Isabel Vieitez Martínez*

Pooja Sripad*

* Population Council.

Coordinación del proyecto, recolección y análisis de datos, redacción y revisión de informe:

Esperanza Basurto-Alcalde, consultora.

Recolección de datos, análisis de datos, y redacción de informe:

Natalia Polgovsky-Ezcurra, consultora.

Recolección de datos (personas pasantes y voluntarias):

Arturo Alamilla, entrevistador. Ana Regina Nájera, entrevistadora. Ximena Nateras, entrevistadora. Iván Coria, entrevistador. Gemma Hernández, entrevistadora.

\section{Corrección de estilo:}

Claudia Martínez.

\section{Ilustración y diseño editorial:}

Valeria García.

\section{Agradecimientos:}

Agradecemos el esfuerzo realizado por el equipo de investigación, sobre todo a personas pasantes y voluntarias, así como la gran disposición que las personas entrevistadas tuvieron para compartirnos su experiencia, en pleno inicio de la pandemia causada por el virus SARS-CoV-2, y por su compromiso constante con las mujeres migrantes y otras poblaciones en movilidad.

\section{Aviso legal:}

Se permite la reproducción total o parcial de esta publicación para fines educativos, de investigación y cualquier otro fin libre de lucro, siempre y cuando se cite la fuente.

\section{Cita sugerida:}

Larrea-Schiavon Silvana, Vázquez-Quesada Lucía, Basurto-Alcalde Esperanza, Polgovsky Natalia, Vieitez Isabel, and Sripad Pooja. 2021. "Atención de la salud sexual y reproductiva de mujeres migrantes: Un mapeo de actores de la sociedad civil en Chiapas, México. 2020." México: Population Council. 


\section{HALLAZGOS PRINCIPALES}

- Chiapas es la principal puerta de entrada terrestre al país para la población en situación de movilidad.

- De acuerdo con datos de la Red de Documentación de las Organizaciones Defensoras de Migrantes (REDODEM), en Chiapas se concentra el mayor porcentaje de agresiones y vividas por la población migrante.

- En las entrevistas se mencionó que aproximadamente $70 \%$ de las mujeres entran a Chiapas a través de la zona selvática de Petén, sufriendo la mayoría de ellas violencia sexual en esta región.

-Se realizó un total de 31 entrevistas a organizaciones de la sociedad civil (OSCs), de las cuales 15 dan alojamiento (10 albergues y 5 refugios).

- El acompañamiento, canalización y consejería en torno al parto es el servicio más ofrecido a las mujeres por las organizaciones y albergues entrevistados. Un 19.3\% de las organizaciones tiene la posibilidad de dar atención directa al parto, en su mayoría por contar con parteras tradicionales.

- La interrupción del embarazo es la atención en salud sexual y reproductiva (SSyR) menos recurrente en la difusión, consejería, canalización y acompañamiento de todos. Un $29 \%$ de las organizaciones tiene la posibilidad de llevarlo a cabo por sus propios medios.

- Las barreras que con mayor frecuencia se mencionan son la discriminación, racismo, xenofobia, costos económicos de los servicios de SSyR y escasez de medicamentos, insumos y acceso a exámenes.

- La COVID-19 ha incrementado las barreras de acceso a la SSyR, reduciéndola a la atención a partos. No hay acceso a atención prenatal ni a estudios diagnósticos para problemas ginecológicos que pueden generar complicaciones.

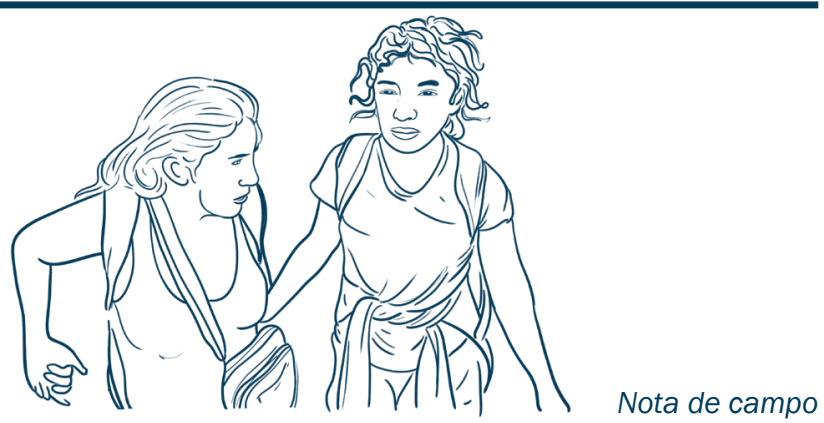

No es lo mismo migrar que migrar siendo mujer.

- Organización, Chiapas
- El acompañamiento directo, junto a la incidencia política, han sido estrategias desarrolladas por las organizaciones y albergues para facilitar el acceso de las mujeres migrantes a los servicios de salud públicos.

\section{INTRODUCCIÓN}

Chiapas colinda en 655 kilómetros con Guatemala, siendo la principal vía de entrada de aquellas personas en movilidad desde Centroamérica y uno de los estados del país donde hay mayor presencia de personas migrantes. Es, asimismo, donde se reciben el mayor número de solicitudes de asilo o refugio de la República mexicana. La población migrante en tránsito proviene sobre todo de Honduras, El Salvador, Guatemala y Haití. Además, dentro de las naciones identificadas por la Matriz de Seguimiento al Desplazamiento de la Organización Internacional para las Migraciones (OIM) un $8 \%$ era originaria de Camerún, $6 \%$ de Cuba, $6 \%$ de India, $3 \%$ de Nicaragua, y un $1 \%$ de países como Yemen, Nepal, Pakistán y Bangladesh (OIM, 2019). Con base en datos de la Unidad de Política Migratoria, Registro e Identidad de las Personas (UPMRIP) de la Secretaría de Gobernación de México, de eneroajuliotanto de 2019 como $2020,42.5 \%$ de los eventos de personas extranjeras presentadas ante la autoridad migratoria mexicana sucedió en Chiapas, ocupando el primer lugar en el país (UPMRIP, 2020).

Por otro lado, es el estado donde se tiene registro del mayor número de agresiones hacia la población migrante. 30.7\% de los actos de violencia registrados en 2018 por la Red de Documentación de las Organizaciones Defensoras de Migrantes (REDODEM, 2019) ocurrieron en Chiapas. En el informe Sin Salida, de Médicos sin Fronteras (MSF), se menciona que la violencia sexual ha sido identificada especialmente en la zona sur del país (como Chiapas y Tabasco). $22.5 \%$ de las mujeres entrevistadas en los puntos de atención de MSF habían sido acosadas sexualmente,12.8\% tuvieron una relación sexual no deseada y $10 \%$ reconocieron haber sido forzadas a tener relaciones sexuales a cambio de algo (MSF, 2020).

Para el caso de Chiapas, no se identificó ningún plan, programa, reglamento, leyes estatales o acuerdos que hicieran referencia directa al derecho al acceso a la salud de la población migrante en el estado. Con respecto al acceso a un aborto seguro, el estado aprobó una reforma constitucional para proteger la vida dese el momento de la 
concepción. Sin embargo, el aborto sigue siendo legal por tres causales: violación, riesgo para la vida de la mujer y malformaciones congénitas (Andar, 2020).

\section{OBJETIVO Y METODOLOGÍA}

El presente estudio se enfocó en identificar si las organizaciones de la sociedad civil brindan los servicios de difusión de información, consejería, canalización, acompañamiento y atención directa a mujeres migrantes en tránsito por México en temas referentes a métodos anticonceptivos, anticoncepción de emergencia, interrupción del embarazo, pruebas de embarazo, atención prenatal, atención del parto, atención del postparto, salud menstrual, infecciones de transmisión sexual (ITS) y atención a sobrevivientes de violencia sexual.

Para alcanzar dicho objetivo se hizo una búsqueda dirigida de organizaciones que trabajaran en temas de SSyR y/o con mujeres migrantes en siete estados claves en la ruta migratoria en la República Mexicana: Chiapas, Tabasco, Veracruz, Oaxaca, Ciudad de México, Chihuahua y Baja California. Se identificó a un actor clave dentro de cada organización y se realizó una entrevista telefónica semiestructurada (para más información ver el reporte general del proyecto). En este resumen ejecutivo se presentan los resultados para el estado de Chiapas.

\section{RESULTADOS}

\section{Perfil de las organizaciones entrevistadas}

Por búsqueda dirigida se identificó un total de 62 organizaciones que cumplían con los criterios de inclusión, de las cuáles se logró entrevistar a 31 (50\%): 9 albergues y refugios, 17 organizaciones de la sociedad civil y 5 organizaciones internacionales. De éstas, 22 cuentan con trabajo a nivel local (70.9\%), 1 a nivel nacional (3.2\%) y 8 son organizaciones internacionales (25.8\%). 21 OSCs se autodefinen como laicas (67.7\%) y 10 como feministas o parte del movimiento feminista (32.2\%).

Las organizaciones entrevistadas se encuentran localizadas en los siguientes municipios ${ }^{1}$ : Arriaga $(n=1)$, Comitán $(n=2)$, Frontera Comalapa $(n=1)$, Huixtla $(n=1)$, Las Margaritas $(n=1)$, Mapastepec $(n=1)$, Palenque $(n=4)$, Salto del Agua $(n=1)$, San Cristóbal de las Casas $(n=7)$, Tapachula $(n=12)$, Tonalá ( $n=1)$ y Tuxtla Gutiérrez $(n=2)$ (ver Gráfica 1).
Gráfica 1. Porcentaje de organizaciones con base en el municipio donde trabajan. Chiapas, 2020.

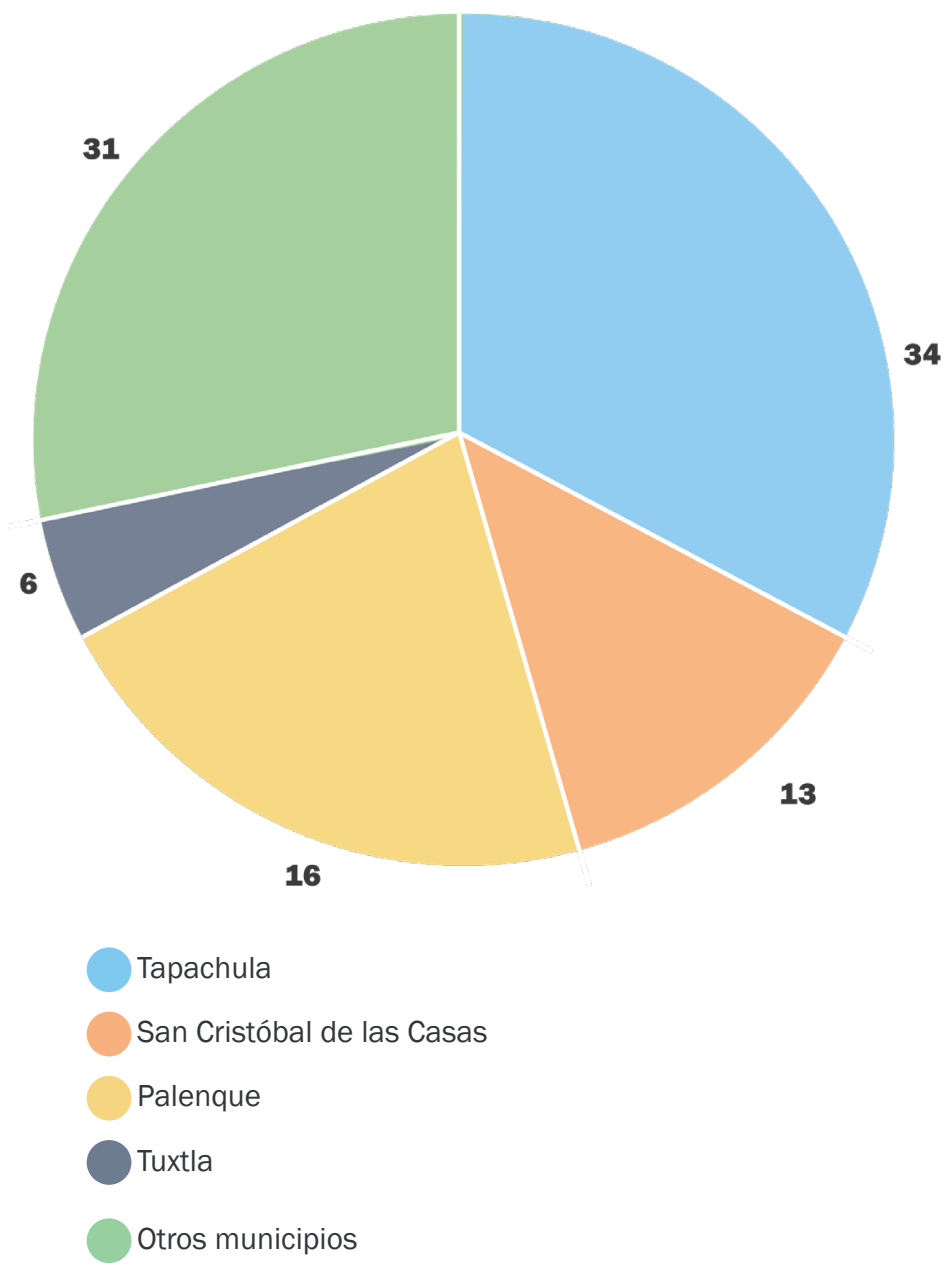

Servicios brindados para la atención de la SSyR de las mujeres migrantes

Del total de organizaciones entrevistadas en Chiapas, 19 (61.2\%) difunden información, 23 (74.1\%) dan consejería, 29 (93.5\%) canalizan casos, 26 (83.8\%) acompañan a las mujeres durante la atención y 12 (38.7\%) brindan atención directa en alguno de los temas mencionadas.

Cuando se hace el cruce entre tipo de servicio (difusión de información, consejería, atención directa, canalización y referencia y acompañamiento) y temática de SSyR en la cual se ofertó este servicio, se identifica que los principales temas sobre los cuales se difundió información en las organizaciones fueron métodos anticonceptivos, atención a mujeres que han vivido violencia sexual, ITS y atención prenatal. Con respecto a los principales temas sobre los cuáles se brindó consejería, se identificaron los de cuidados maternos (atención prenatal, atención del parto y atención postparto), pruebas de embarazo, métodos anticonceptivos y atención a sobrevivientes de violencia sexual. La atención

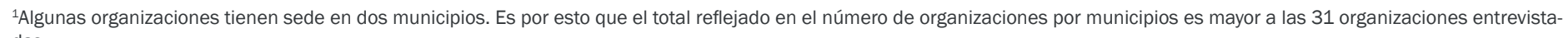


directa brindada por las organizaciones se concentró principalmente alrededor de la atención a sobrevivientes de violencia sexual, métodos anticonceptivos, pruebas de embarazo y salud menstrual. La canalización y referencia se brindó para todos los temas por más del 70\% de las organizaciones, excepto para la interrupción del embarazo, donde $54.8 \%$ de las organizaciones entrevistadas dijeron canalizar a las mujeres a otras OSCs para la interrupción. Por último, el acompañamiento se dio en casi todos los temas por más del $70 \%$ de las organizaciones, a excepción de anticoncepción de emergencia e interrupción del embarazo.
La atención directa a salud menstrual se limitó a facilitar el acceso a toallas higiénicas. Es relevante mencionar que 29\% de las organizaciones mencionó dar atención directa para informar sobre la interrupción del embarazo y 32.2\% para anticoncepción de emergencia. Para el caso de métodos anticonceptivos, los preservativos masculinos fueron el método al cual se tuvo mayor acceso (ver Tabla 1).

Tabla 1. Porcentaje de organizaciones que ofrecen servicios de salud sexual y reproductiva, por tipo de servicio ofertado. Chiapas, 2020.

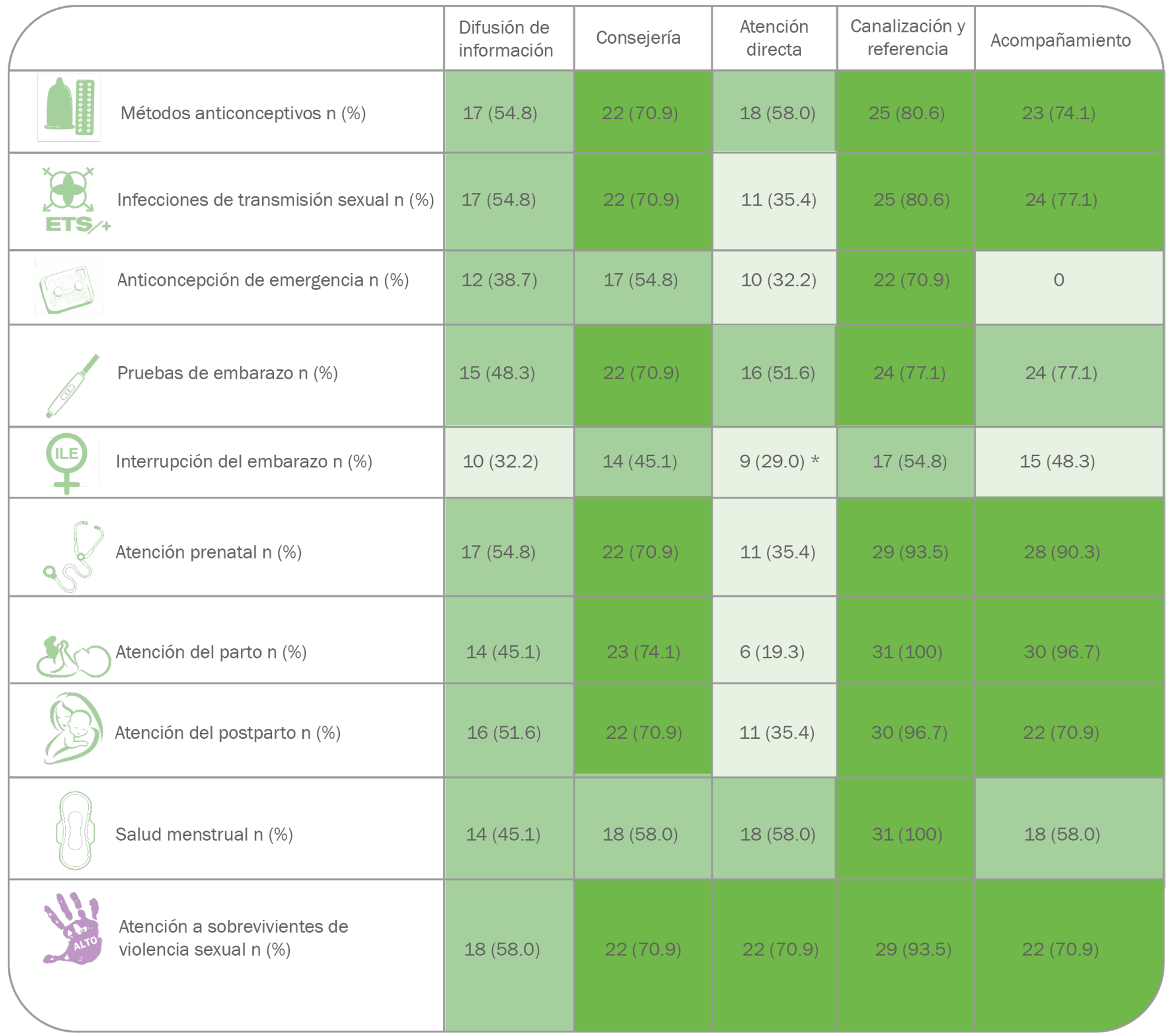

Nota: verde obscuro, el servicio es ofertado por $>70 \%$ de las organizaciones entrevistadas; verde intermedio, el servicio es ofertado por entre $36 \%$ - $69 \%$ de las organizaciones entrevistadas; verde claro, el servicio es ofertado por $<36 \%$ de las organizaciones entrevistadas.

* La atención directa en el tema de interrupción del embarazo consiste en brindar información pública amplia sobre el marco legal (particularmente de la NOM-046), los métodos recomendados por la Organización Mundial de la Salud, y los requisitos para acceder a un servicio legal. Las OSCs no realizan los procedimientos, ni promueven la interrupción del embarazo - pues realizarlo es decisión de la mujer embarazada y de quien ella quiera involucrar al tomarla-. Se diferencia de "difusión de información” en tanto los temas cubiertos son más amplios en esta categoría. 
El tema sobre el cual se tiene mayor apertura para hablar y discutir es la atención del parto. Las organizaciones con parteras tienen la capacidad de dar atención directa a partos y reportaron la preferencia de las mujeres migrantes por esta opción y no por atención en unidades de salud. El resto de las oSCs canalizaron a las mujeres a unidades de salud y/o las acompañaron durante el proceso. Por último, existe un número importante de organizaciones que canalizan, acompañan y dan atención directa a sobrevivientes de violencia sexual. La atención directa que se ofreció es principalmente atención psicológica y, en un número reducido de casos, el acceso a profilaxis postexposición para VIH. La dificultad señalada por las organizaciones en este tema es que en pocas ocasiones las mujeres migrantes informan de lo ocurrido, por lo que varias OSCs han desarrollado protocolos de detección y actuación.

Según relataron las personas entrevistadas, muchas mujeres migrantes, antes de comenzar su viaje, deciden iniciar un método anticonceptivo para evitar embarazos no deseados, conscientes de la violencia sexual que pueden vivir durante el trayecto. En otros casos son sus parejas, los hombres que las acompañan, los que deciden sobre el uso del método anticonceptivo. En mujeres migrantes provenientes de África y Haití se reportan altos índices de embarazos no deseados en el trayecto, sin que se especificara un motivo; pero puede estar asociado a una mayor vulnerabilidad a agresiones sexuales o razones culturales, entre otros factores.

Cuando observan que no pueden dar la atención directa, las organizaciones recurren a la canalización hacia otras oSCs, al sistema de salud público o a médicos y consultorios particulares, no obstante, las posibilidades de atención dependen en cierta medida de la presencia de personal de salud en la organización. De un total de 31 organizaciones entrevistadas, 8 cuentan con personal médico de base (26\%), 11 cuentan con otro personal de salud (enfermería, psicología y parteras) pero no cuentan con personal médico (35\%) y 12 no cuentan con ningún tipo de personal de salud (39\%) (ver Gráfica 2).

\section{Principales barreras para la atención en} SSyR a mujeres migrantes

En las entrevistas con organizaciones de la sociedad civil en Chiapas se identificaron múltiples barreras para que las mujeres migrantes pudieran acceder a los servicios de SSyR durante su estancia o tránsito por el estado. En el caso del presente estudio, las barreras para el acceso a la salud fueron clasificadas en cuatro categorías:
Gráfica 2. Porcentaje de organizaciones que cuentan con personal de salud en sus instalaciones. Chiapas, 2020.

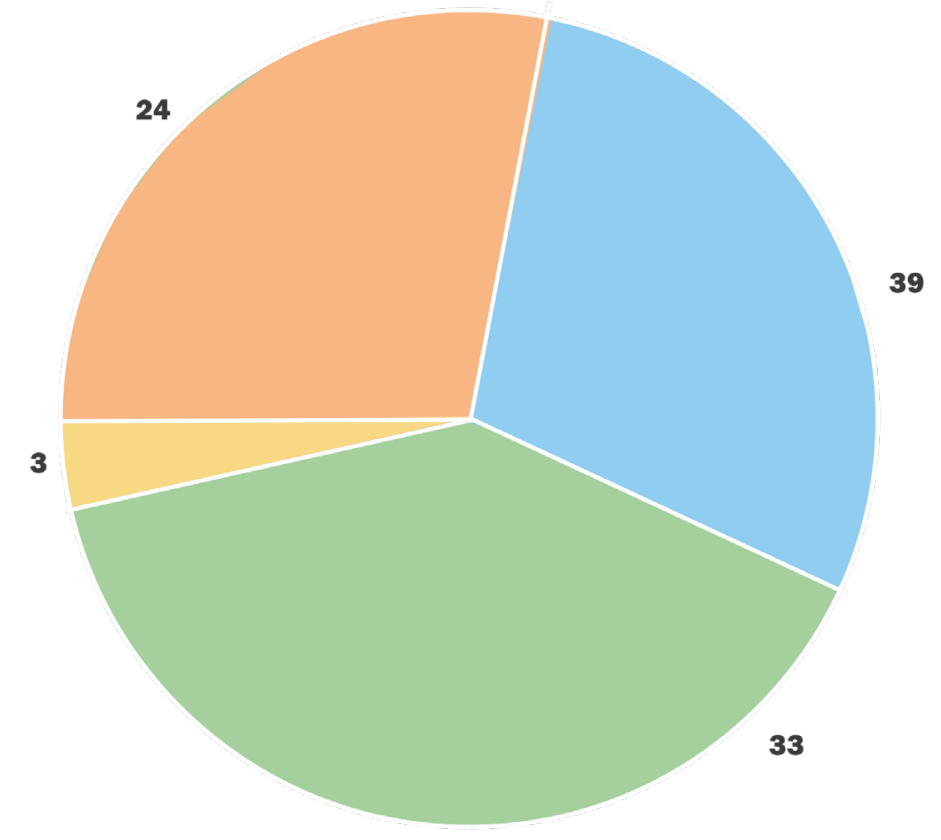

Sin personal de salud

Médica/o de base

Médica/o voluntaria/o

Otra/os profesionales de la salud

1. Normativas: relacionadas con la legislación, políticas y normatividad que aplican tanto a la población migrante (marco restrictivo para la atención), como a las instituciones de salud;

2. Institucionales: impedimentos estructurales de la institución de salud que brinda o debería brindar los servicios;

3. Organizacionales: impedimentos estructurales de la organización de la sociedad civil que brinda los servicios; e

4. Individuales: obstáculos derivados de la condición socioeconómica, cultural y subjetiva de las personas migrantes (ver Tabla 2).

Las principales barreras normativas percibidas por las organizaciones se vinculan con 1) la interrupción del embarazo, ya que en Chiapas el aborto está permitido solamente bajo tres causales, que son por violación, riesgo para la vida de la mujer y malformaciones congénitas (Andar, 2020) -y no siempre las mujeres, profesionales de la salud y otras autoridades están informadas al respecto-. Otras barreras identificadas fueron 2) el cambio del Seguro Popular al Instituto de Salud para el Bienestar (Insabi) y la ausencia de reglas de operación claras de este último; así como 3) la ausencia de políticas públicas que reconozcan 
las necesidades específicas de las mujeres migrantes en el estado.

Con respecto a las instituciones de salud, $64.5 \%$ de las organizaciones mencionaron que existe una falta de acceso a atención de calidad en SSyR en el estado. Las principales barreras institucionales mencionadas en las entrevistas fueron 1)la falta de insumos (principalmente medicamentos y material de curación) en las unidades de salud. En municipios como Palenque hay coincidencia en que los materiales necesarios para ser atendidas, como pinzas o instrumental médico básico, los tiene que llevar consigo la paciente para recibir el servicio. En cuanto a métodos anticonceptivos las respuestas varían, hay organizaciones que manifestaron sí tener acceso a preservativos y a algunos métodos inyectables a través de instituciones de salud pública, mientras que otras señalan que no. También se hizo referencia a la ausencia de pruebas de detección de ITS y a medicamentos antirretrovirales. En estos casos, es frecuente que se soliciten traslados, ya que solo las unidades de algunos municipios cuentan con estos medicamentos. Además, se señaló 2) la limitada infraestructura de las unidades de salud en algunos municipios; 3) la carencia de personal médico, mencionada en 1 de cada 4 entrevistas. Según los testimonios recopilados, esto implica una saturación en los centros de salud y retrasos en la atención. El personal médico atiende a más personas de las que corresponden con su capacidad. Las plantillas están incompletas en varios turnos, sin que haya médicos especialistas, incluyendo ginecólogas o ginecólogos. Se denunció además que una de las áreas donde tienen más retrasos dentro de los servicios públicos es la de ginecología y obstetricia. También se identificó 4) la necesidad de capacitación y sensibilización de personal de salud en temas como derechos humanos y personas migrantes, en específico sobre mujeres migrantes, tanto para el personal de salud como para las autoridades locales; 5)la presencia de actitudes discriminatorias, misóginas y machistas del personal de salud hacia las mujeres migrantes, lo cual llevó en ciertas ocasiones a la revictimización (en casos de violencia sexual) y violencia obstétrica durante la atención del parto; 6)la presencia de objetores de conciencia en las unidades de salud que limitó el acceso a los servicios de interrupción del embarazo; y 7) la necesidad de que las mujeres migrantes acudieran acompañadas a las unidades de salud para ser atendidas. Hablando específicamente de métodos anticonceptivos y anticoncepción de emergencia, se mencionó 8)la falta de difusión de los derechos y servicios existentes, así como la resistencia del personal de salud a una promoción más amplia de los métodos de planificación familiar, mucho más para las mujeres migrantes. Del mismo modo, se alude a la gran dificultad existente para acceder a anticoncepción de emergencia en 2 de los municipios con mayor población del estado.

Las barreras relativas a las organizaciones de la sociedad civil están relacionadas con 1)la falta de insumos médicos en sus instalaciones (medicamentos, insumos para la salud menstrual, métodos anticonceptivos y material médico), 2) la falta de personal de salud (principalmente personal médico) en las organizaciones, y 3)un bajo conocimiento del personal de las OSCs en temas de SSyR.

Por último, las barreras individuales identificadas fueron 1) la dificultad económica, $64.5 \%$ de las organizaciones entrevistadas hicieron referencia explícita a que la falta de dinero dificulta o impide que las mujeres puedan acceder a los servicios, ya que no tienen recursos para medicamentos, traslados, estudios (ultrasonidos), consultas privadas o los insumos que solicitan algunos hospitales para atender los partos o colocar un dispositivo intrauterino; 2)el idioma, sobre todo para mujeres de pueblos indígenas y para mujeres haitianas; 3)la edad, ya que se mencionó que se atiende con mayor facilidad a las mujeres migrantes de mayor edad en comparación con las más jóvenes; 4)la presencia de miedo a acudir a una unidad de salud, sobre todo si se asocia a una experiencia previa de malos tratos y discriminación o si la persona fue violentada por una autoridad, así como el temor a que el personal de salud avise a las autoridades migratorias de su situación indocumentada y, en el caso de mujeres víctimas de trata de personas, el miedo a ser encontradas por sus captores si acuden a las unidades de salud; 5)el desconocimiento sobre los cuidados necesarios, la importancia del tiempo y la ubicación de lugares donde atenderse -varias organizaciones hicieron referencia a que las mujeres migrantes aguantan el dolor y no lo cuentan-. Algunas organizaciones manifestaron que no hay cultura de autocuidado entre las mujeres y que hay desconocimiento

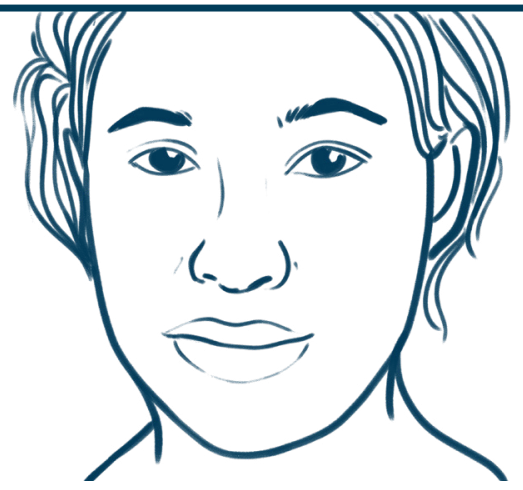

Nota de campo Incluso en atención privada, como Farmacias Similares, si ven que son migrantes no les atienden.

- Albergue, Chiapas 
sobre la importancia de la SSyR. Finalmente, 6) la religión, ya que las mujeres con valores religiosos cristianos son menos proclives a interrumpir un embarazo o hablar de la SSyR en general. Específicamente en relación con la interrupción del embarazo, se especificó que las creencias religiosas están vinculadas a la experimentación de miedo, duda, culpa o arrepentimiento por parte de las mujeres.

Dentro de la atención integral a la SSyR, la interrupción del embarazo es la que más obstáculos encuentra, siendo todavía un tema controvertido, culturalmente estigmatizado e ilegal por voluntad propia en el estado. Difícilmente se puede acceder a una interrupción en las causales por las que es legal, como la violencia sexual. Esto está vinculado en algunos casos a objeciones de conciencia, pero también a desconocimiento de la legislación vigente. Se reportó además que hay una cuestión de lucro en torno a la objeción de conciencia, pues hay médicos/as que rechazan realizar la interrupción en el sistema de salud público, pero no en el privado.

La atención a mujeres que vivieron violencia sexual también presentó obstáculos para ser atendida. Según las organizaciones entrevistadas hay omisiones en la atención. Por ejemplo, a las mujeres migrantes no se les da acceso a atención psicológica y se reportaron casos de negaciones a la interrupción en caso de embarazos derivados de la agresión. También se informó de casos en los que la atención es revictimizante y en los que se criminaliza a las mujeres que quieren iniciar un proceso de interrupción por esta causa.

A ello se suma que en ocasiones se da aviso al Ministerio Público sin consentimiento de la persona. Además, ha de considerarse la dificultad que implica hablar de una agresión en el contexto planteado y con la presencia en algunos casos de sentimientos de culpa por lo ocurrido. Varias de las OSCs

Tabla 2. Resumen de principales barreras para que las mujeres migrantes accedan a los servicios de salud en Chiapas, 2020.

\begin{tabular}{|c|c|}
\hline Clasificación & Principales barreras \\
\hline Normativas & $\begin{array}{l}\text {-Normatividad del estado relativa a la interrupción del embarazo } \\
\text {-Implementación del Insabi y desaparición del Seguro Popular } \\
\text {-Ausencia de políticas públicas que reconozcan las necesidades específicas de las } \\
\text { mujeres migrantes }\end{array}$ \\
\hline Institucionales & $\begin{array}{l}\text {-Falta de insumos (medicamentos, métodos anticonceptivos y material médico) } \\
\text {-Limitada infraestructura de algunas unidades de salud } \\
\text {-Falta de personal médico, sobre todo especialistas } \\
\text {-Necesidad de capacitación y sensibilización del personal de salud y de las } \\
\text { autoridades locales } \\
\text {-Presencia de actitudes discriminatorias y misóginas por parte del personal de salud } \\
\text {-Presencia de objetores de conciencia } \\
\text {-Necesidad de acompañamiento a las unidades de salud }\end{array}$ \\
\hline Organizacionales & $\begin{array}{l}\text {-Falta de recursos (económicos e insumos médicos) } \\
\text {-Falta de personal de salud } \\
\text {-Falta de formación en SSyR del personal }\end{array}$ \\
\hline Individuales & $\begin{array}{l}\text {-Dificultades económicas } \\
\text {-Idioma } \\
\text {-Edad } \\
\text {-Miedo } \\
\text {-Desconocimiento } \\
\text {-Religión }\end{array}$ \\
\hline
\end{tabular}


entrevistadas explicaron que las mujeres a menudo no les cuentan que han vivido violencia sexual en su camino.

Por municipios, se señaló a Tapachula como el lugar en el que el racismo y la xenofobia están más marcados, aunado a que enfrenta un gran reto presupuestal pues no se considera a la población en movilidad a la hora de asignar los recursos para salud pública, lo que conduce a una falta de infraestructura, personal, insumos y medicamentos para la población total que necesita ser atendida. Además, en Tapachula se tiene constancia de la prostitución ejercida por mujeres migrantes y la estigmatización que tienen al intentar acceder a servicios de salud.

En Palenque hubo muestras de rechazo ciudadano a las personas migrantes, pudiendo estar relacionadas con incidentes aislados de conductas de migrantes vinculadas a la ingesta de alcohol. Se mencionó que estas actitudes se han exacerbado por el temor al contagio de la COVID-19 y la consideración -no fundamentada- de las personas migrantes como vectores del virus. Además, es un municipio en el que se reporta escasez de recursos y de infraestructura en salud, lo que implicó traslados a Villahermosa, Tabasco para recibir atención en salud especializada.

Por último, algunas organizaciones también han mejorado su vinculación con las autoridades sanitarias y municipales correspondientes a raíz de la llegada de las "caravanas migrantes", pero se reportó que la coordinación entre organizaciones y con las autoridades de salud no es tan estrecha una vez pasada la coyuntura.

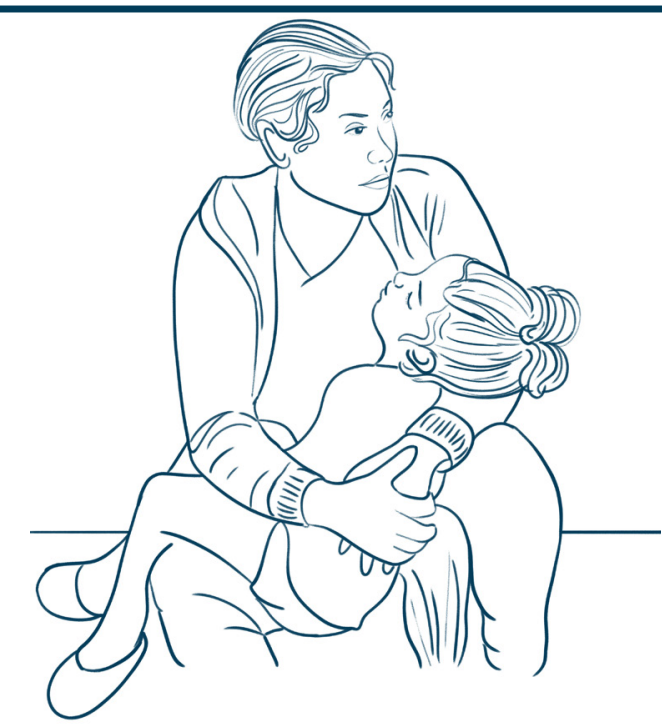

Hay mucho miedo en torno al aborto. Hay tanto critica de la Nota de campo sociedad conservadora como de las autoridades conservadoras.

- Refugio, Chiapas
COVID-19, mujeres migrantes y acceso a servicios de SSYR

En general, y de acuerdo con el reporte de las organizaciones entrevistadas en los siete estados que son parte de este estudio ${ }^{2}$, se identificó que la pandemia por COVID-19 tuvo los siguientes efectos en las OSCs y en la población migrante, particularmente en las mujeres y su acceso a servicios de SSyR:

- Reducción de unidades de salud y servicios disponibles. La reconversión de clínicas y hospitales a Centros COVID y la atención restringida a casos de emergencia, limitó de manera significativa la atención en las unidades médicas, ya sea por retraso o negación de la atención.

- Cierre temporal y/o parcial de albergues. Incluso aquellos que se mantuvieron abiertos dejaron de recibir a personas, ya que las y los migrantes no acudían porque escucharon que estaban cerrados.

- Disminución de recursos en las OSCs para prestar la atención. Con la pandemia hubo una reducción de recursos recibidos a través de donativos, además de tener una baja en el número de personas voluntarias, y del personal en general para brindar servicios y atención, incluyendo a personal médico.

- Permanencia prolongada de migrantes en los lugares, al no poder seguir su viaje. El cierre de oficinas gubernamentales -como la Comar- afectó a aquellas personas que esperaban la resolución a su solicitud de condición de refugiada u otro trámite similar, y les obligó a no salir de la entidad donde iniciaron el procedimiento.

- Afectaciones a la salud mental de las personas migrantes. Incremento de las preocupaciones relacionadas con sus procesos frente a autoridades migratorias, y/o por el confinamiento en albergues.

- Pérdida de trabajos y recursos económicos. Con el cierre de establecimientos comerciales hubo pérdida de empleos que también afectó a personas migrantes, pues se enfentan a la imposibilidad de laborar y tener el ingreso para solventar sus necesidades básicas.

2En este apartado se incluye información general y que se observó para los siete estados: Chiapas, Tabasco, Veracruz, Oaxaca, Ciudad de México, Chihuahua y Baja California. 
- Miedo a asistir a las unidades médicas. Las personas migrantes tienen temor a contagiarse si asisten a las unidades médicas, por lo que retrasan o desisten de la atención.

\section{CONCLUSIONES Y ÁREAS DE OPORTUNIDAD ${ }^{3}$}

Las posibilidades para mejorar la atención de la SSyR de las mujeres son amplias, desde el fortalecimiento el sistema de salud público que garantice el acceso a estos servicios, hasta apoyando a las organizaciones y albergues que están dando acceso o facilitando el mismo. Las organizaciones están dedicando sus recursos a garantizar el acceso a los servicios donde observan que hay vacíos en la atención, o una atención insuficiente, permitiendo el cumplimiento de lo que es un derecho humano. Si bien las OSCs no son responsables garantes de brindar servicios de salud, han demostrado ser clave para contribuir a este propósito. En el campo en que se enfocó el estudio (SSyR de las mujeres migrantes), el rol de las OSCs es particularmente relevante, no sólo por ser muchas veces el primer punto de contacto, sino porque la confianza que ganan con su trabajo les habilita para tratar estos temas -considerados de mayor intimidad-.

\section{El Estado, a través de los distritos sanitarios (antes jurisdicciones sanitarias) y de acuerdo con el Plan Integral} de Atención a la Salud de la Población Migrante (SSA, 2020), tiene la responsabilidad de garantizar que los servicios de SSyR (incluido el aborto legal) se brinden de forma oportuna a las mujeres migrantes, y de llevar a cabo las tareas necesarias (ej. capacitar y sensibilizar al personal que labora en las instituciones) para que brinden la atención con la más alta calidad posible.

Las organizaciones en Chiapas identificaron áreas de oportunidad y responsabilidad en las instituciones, como un adecuado financiamiento para el sector salud (ej. contar con la medicación -gratuita- que recetan, posibilidad de hacer los estudios indicados - ultrasonidos- en la unidad de salud, insumos suficientes o variados en el caso de anticoncepción); mejorar la infraestructura de las unidades de salud; aumentar la contratación de personal médico -sobre todo especialistas-; promover un enfoque sensible y especializado para la atención a población en situación de movilidad entre autoridades de las unidades de salud, e incluir al personal médico y no-médico; e incluir dinámicas efectivas para romper prejuicios sobre esta población. También se sugiere mejorar el sistema de referencia y contrarreferencia entre las unidades médicas; y capacitar al personal de salud sobre el aborto legal en el estado, así como sobre los derechos y responsabilidades en la objeción de conciencia. En Chiapas, las organizaciones identificaron elementos a fortalecer en centros de salud y hospitales específicos (ver Tabla 3).

Las organizaciones que trabajan en Chiapas atienden varios aspectos de la SSyR de las mujeres migrantes (ej. parto, violencia sexual, anticoncepción, VIH). El trabajo con las mujeres parteras es una experiencia que debe rescatarse y fortalecerse por parte de la Secretaría de Salud -sobre todo en lo relativo a la normativa para integrar servicios de partería dentro y fuera de las unidades médicas, y que permitan a las parteras emitir certificados de nacimiento-. La interrupción del embarazo, como en otros estados, es un aspecto de la salud reproductiva menos atendido. La capacidad de responder a las necesidades de las mujeres migrantes se hace mediante el trabajo en articulación, siendo la canalización y referencia -entre OSCs o con las unidades de salud- el recurso más utilizado. Para que la canalización sea efectiva en las unidades de salud, muchas veces las organizaciones deben acompañar a las mujeres migrantes en la búsqueda de atención. En este sentido, formalizar mecanismos de coordinación sustentables es una necesidad que se deriva del reporte de las organizaciones. Sin embargo, en ciertas coyunturas como las "caravanas migrantes", se ha demostrado voluntad política de todas las partes (tanto autoridades de salud -secretaría estatal, jurisdicción sanitaria, directivos de las unidades médicascomo organizaciones) para la articulación en beneficio de las mujeres migrantes. El liderazgo de ACNUR para hacer sostenible el trabajo conjunto y coordinado en los sitios donde tiene oficinas (Palenque y Tapachula) resulta vital en este sentido.

Otra necesidad sentida y expresada al interior de las OSCs en Chiapas para atender las necesidades de SSyR de las mujeres migrantes (tanto albergues, como organizaciones nacionales e internacionales), es el acceso a financiamiento para contratar personal, y apoyar con insumos como anticonceptivos, toallas higiénicas, o medicamentos recetados en los servicios de salud a las mujeres migrantes (o también para mejorar la infraestructura en el caso de los albergues y refugios). Algunas organizaciones han reducido sus servicios debido a dificultades económicas que se agravan con la pandemia. Para cubrir esta brecha, es necesario el apoyo gubernamental estatal y federal, de agencias internacionales, y de personas individuales. Por tanto, se hace un llamado a estos actores a no desestimar el papel que las organizaciones cubren para procurar el bienestar de las personas migrantes, y, en consecuencia, financiar su trabajo. 
A pesar de estas dificultades, dentro de su contexto y posibilidades, las organizaciones de la sociedad civil pueden, entre otras cosas, ayudar a monitorear las necesidades de SSyR de esta población; brindar información/educación de calidad sobre la SSyR (con base en las necesidades identificadas), y sobre los pasos para ejercer su derecho a la salud; identificar (y educar también sobre) síntomas y señales de riesgo que indican que una mujer debe de ser atendida de urgencia; y establecer mecanismos formales de referencia a unidades de salud correspondientes con el tema a tratar.

Incluir información que visibilice la SSyR en los materiales que generan las organizaciones puede facilitar que las mujeres le den también importancia. Las organizaciones entrevistadas tienen la experiencia de hacer llegar información en idiomas distintos al castellano (ej. lenguas indígenas y creole) en trípticos o cápsulas informativas para redes sociales, así como información para las personas con baja alfabetización a través de radios comerciales, comunitarias, o materiales gráficos puntuales.

Otra área de oportunidad es la de contar con un sistema de información que les permita el monitoreo de la población a la que atienden y los problemas de salud (en general y en particular, SSyR) presentados -entre otras temáticas relacionadas con el respeto a sus derechos humanos-. La finalidad del monitoreo es la de sistematizar e identificar las necesidades más apremiantes de esta población y cómo se van transformando en el tiempo. Esto ayudará a las organizaciones a contar con información para hacer

Tabla 3. Elementos para fortalecer en centros de salud y hospitales específicos. Chiapas, 2020.

\begin{tabular}{|c|c|c|}
\hline Unidad de salud & Municipio & Puntos a fortalecer \\
\hline $\begin{array}{l}\text { Centro de Salud } \\
\text { Reproductiva }\end{array}$ & Comitán & $\begin{array}{l}\text { Desabasto de medicamentos, especialmente de métodos } \\
\text { anticonceptivos. Ausencia de personas en atención de } \\
\text { SSyR, y visibilidad del área. Trato discriminatorio de las } \\
\text { trabajadoras sociales. }\end{array}$ \\
\hline Hospital de la Mujer & San Cristóbal de las Casas & $\begin{array}{l}\text { Carece de un área especializada para mujeres migrantes } \\
\text { que sea no discriminatoria, que conozcan más los } \\
\text { contextos migratorios y el tipo de situaciones que ellas } \\
\text { afrontan (ej. trata de personas). Falta de personal, camas } \\
\text { e insumos, incluidos los necesarios para atender partos. }\end{array}$ \\
\hline Hospital de la Mujer & Tapachula & $\begin{array}{l}\text { Estigma, mal trato y discriminación. No brinda atención si } \\
\text { no es una emergencia, dificultad para acceder a la } \\
\text { interrupción del embarazo. No garantiza el acceso a } \\
\text { ultrasonidos. }\end{array}$ \\
\hline Hospital General & Palenque & $\begin{array}{l}\text { Poca capacidad para la atención, limitada infraestructura } \\
\text { y poco personal médico para atender a toda la población. } \\
\text { No cuenta con especialistas suficientes. Falta de medica- } \\
\text { mentos e insumos. }\end{array}$ \\
\hline Hospital General & Tapachula & $\begin{array}{l}\text { Falta de insumos, personal, así como capacitación y } \\
\text { sensibilización de éste en derechos de las personas en } \\
\text { situación de movilidad. Requieren intérpretes y generar } \\
\text { información en otros idiomas. No garantizan el acceso a } \\
\text { ultrasonidos, rayos X, pruebas ITS y exámenes de labora- } \\
\text { torio. Requieren un mejor sistema de contrareferencia y } \\
\text { facilitar que llegue el medicamento de la preclamsia a los } \\
\text { centros de salud. }\end{array}$ \\
\hline Hospital General & Tuxtla & $\begin{array}{l}\text { Falta de insumos de todo tipo, incluyendo medicamentos. } \\
\text { Presentan tardanza en dar la atención y requieren } \\
\text { capacitación para la no discriminación. }\end{array}$ \\
\hline
\end{tabular}


incidencia con las instituciones de salud gubernamentales, y recomendar la priorización de esfuerzos y recursos en el corto, mediano, y largo plazo. El sistema de información se puede generar también con el apoyo de organizaciones de la sociedad civil nacionales y/o internacionales.

las organizaciones a contar con información para hacer incidencia con las instituciones de salud gubernamentales, y recomendar la priorización de esfuerzos y recursos en el corto, mediano, y largo plazo. El sistema de información se puede generar también con el apoyo de organizaciones de la sociedad civil nacionales y/o internacionales. Con el fin de hacerse de recursos para la creación de este sistema de información seguro y compartido entre las redes de apoyo a migrantes, se puede insistir a través de la incidencia con quienes financian trabajo en este campo.

A pesar de las limitaciones del estudio (como contar sólo con el reporte de las OSCs, y no con la perspectiva ni de las mujeres ni del sistema de salud local), la investigación muestra importantes áreas de oportunidad para lograr la atención a la SSyR de las mujeres migrantes. Investigaciones futuras deberán de indagar directamente sobre las necesidades de SSyR de mujeres, adolescentes y niñas, y las necesidades del sector salud para poder prestar la atención. La investigación operativa puede mejorar el funcionamiento de las OSCs como puentes para acercar a las mujeres a los servicios de salud, y apoyar a las unidades médicas a mejorar sus protocolos, así como a entender por qué ciertos esfuerzos - de capacitación, por ejemplo-, pueden no estar dando el resultado esperado.

La salud sexual y reproductiva de las mujeres migrantes en tránsito por México es un derecho. Tomar conciencia de que las necesidades de salud de las mujeres son minimizadas, incluso por ellas mismas, es uno de los retos hacia la equidad de género. Facilitar y garantizar el cuidado a su salud forma parte de los esfuerzos para lograr el respeto a los derechos humanos.

Agradecemos el esfuerzo realizado por el equipo de investigación durante los meses de trabajo, así como la gran disposición que las personas entrevistadas tuvieron para compartirnos su experiencia en medio de la pandemia de SARS-CoV-2, y por su compromiso constante con las mujeres migrantes.
El presente estudio es evidencia de que las organizaciones de la sociedad civil locales e internacionales trabajan en primera línea para defender, promover, y apoyar el alcance de los derechos de las personas migrantes -incluso coadyuvando en el acceso a servicios de salud sexual y reproductiva-. El reconocimiento a ellas y a su labor va acompañado de un llamado a la solidaridad para impulsar su financiamiento y generar condiciones que permitan su desarrollo y el de sus colaboradoras/es.

En asociación con los ministerios de salud nacionales y otras agencias gubernamentales en África subsahariana, el sur de Asia y América Latina, las y los investigadores del Population Council están Ilevando a cabo investigaciones de salud pública y ciencias sociales sobre el COVID-19 para producir evidencia relevante y oportuna para apoyar a la formulación de políticas para el control de la propagación del coronavirus, la evaluación de la eficacia de las medidas de prevención y mitigación y la evaluación de los impactos sanitarios, sociales y económicos a largo plazo de la pandemia.

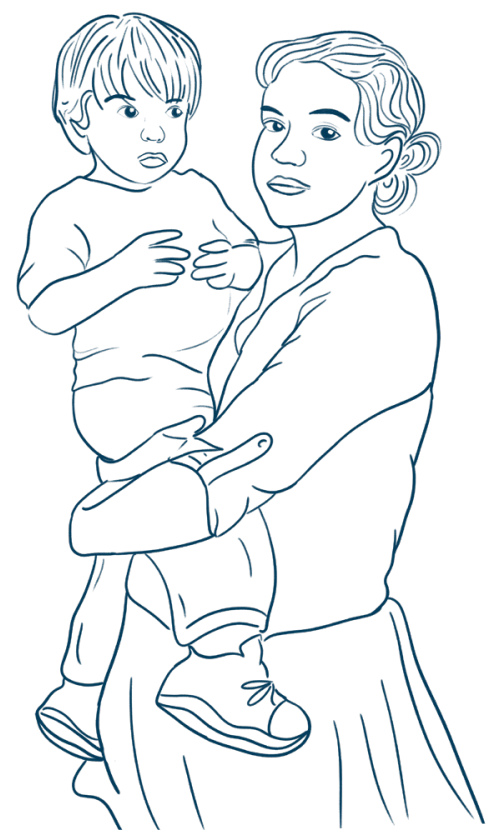

Population Council México. 2021. Atención de la salud sexual y reproductiva de mujeres migrantes: Un mapeo de actores de la sociedad civil en México. Resultados principales para el estado de Chiapas. Ciudad de México, México: Population Council. 